\title{
A monolayer of three-level quantum $\Lambda$-emitters: A perspective system from the viewpoint of nonlinear optical dynamics and nanophotonics
}

\author{
Igor Ryzhov ${ }^{1, *}$, Ramil Malikov², Andrei Malyshev ${ }^{3,4}$, and Victor Malyshev ${ }^{1,5}$ \\ ${ }^{1}$ Herzen State Pedagogical University of Russia, 191186 St.-Petersburg, Russia \\ ${ }^{2}$ Akmullah State Pedagogical University of Bashkortostan, 450000 Ufa, Russia \\ ${ }^{3}$ GISC, Departamento de Física de Materiales, Universidad Complutense, E-28040 Madrid, Spain \\ ${ }^{4}$ Ioffe Physical-Technical Institute, 194021 St.-Petersburg, Russia \\ ${ }^{5}$ University of Groningen, Z IAM, 9747 AG Groningen, The Netherlands
}

\begin{abstract}
The nonlinear optical response of a monolayer of regularly spaced three-level quantum emitters with a doublet in the ground state is studied theoretically. It is found that the system demonstrates multistability, self-oscillations and dynamical chaos. In a certain frequency range, the monolayer operates as a perfect bistable mirror.
\end{abstract}

We perform a theoretical study of the optical response of a monolayer of regularly spaced three-level quantum emitters (QEs) with a doublet in the ground state ( $\Lambda$-type $Q E)$. The total (retarded) dipole-dipole interaction of QEs is taken into account. This interaction provides a positive feedback which, interplaying with the immanent QE's nonlinearity, results in multistability, self-oscillations and dynamical chaos of the response. In a certain frequency range, the monolayer operates as a perfect nanometric bistable reflector.

The monolayer is assumed to undergo an action of a CW external field of a Rabi amplitude $\Omega_{0}$ and frequency $\omega_{0}$, which is quasi-resonant with the QE's allowed transitions. A QE is modelled as a three-level $\Lambda$-type quantum system with the excited state $|3\rangle$, and a doublet $|1\rangle$ and $|2\rangle$ in the ground states. Only the optical transitions $|1\rangle \leftrightarrow|3\rangle$ and $|2\rangle \leftrightarrow|3\rangle$ are dipole-allowed. They are characterized by the transition frequencies $\omega_{31}$ and $\omega_{32}$, and spontaneous decay constants $\gamma_{31}$ and $\gamma_{32}$. The relaxation within the doublet with the splitting $\Delta_{21}$ is described by the constant $\gamma_{21}$.

The optical dynamics of a QE is governed by the $3 \times 3$ density matrix. The field $\Omega$, acting on a given $\mathrm{QE}$ in the monolayer, represents a sum of the external field $\Omega_{0}$ and the field produced by all other QEs in place of the given one. The near-zone (far-zone) part of the QEQE interaction gives rise to a dynamic shift of the transition frequencies $\omega_{21}$ and $\omega_{32}$ (collective radiative relaxation of QEs), depending on the population difference of corresponding transitions [1,2], governing by the constants $\Delta_{\mathrm{L}}$ (shift) and $\gamma_{\mathrm{R}}$ (relaxation).

In Fig. 1, we present the result of calculations performed for the case when the external field is tuned into the resonance with the transition $|1\rangle \leftrightarrow|3\rangle\left(\Delta_{31}=\omega_{31}-\omega_{0}=0\right)$. Panel (b) shows the steady-state solution $|\Omega|$-vs- $\left|\Omega_{0}\right|$ for the set of parameters [2,3] given in the figure

\footnotetext{
*Corresponding author: igoryzhov@yandex.ru
} 


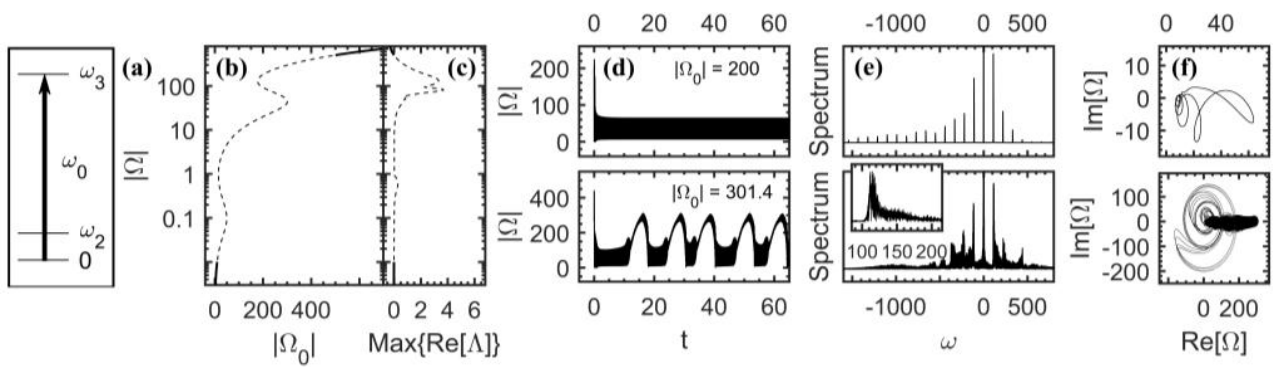

Fig. 1. (a) - Scheme of QE excitation. (b) - The monolayer's steady-state response. (c) - The real part of the major Lyapunov exponent. Solid (dashed) parts indicates stable (unstable) of the solution. (d), (e) and (f) - Respectively, dynamics, Fourier spectra and phase space trajectories of the system calculated for the field magnitudes $\left|\Omega_{0}\right|$ shown in panel (d). Parameters of calculations are: $\Delta_{21}=100$, $\gamma_{\mathrm{R}}=100, \Delta_{\mathrm{L}}=1000, \gamma_{21}=0.01$. All quantities are given in units of $\gamma_{31}$.

caption. As is seen, $|\Omega|$ turns out to be a multi-valued function of $\mid \Omega_{0}$ The stability of different parts of the solution has been explored analyzing the Lyapunov's exponents $\Lambda_{\mathrm{k}}(\mathrm{k}=1,2 \ldots 8)$. The maximal real part of $\left\{\Lambda_{\mathrm{k}}\right\}, \operatorname{Max}\{\operatorname{Re}[\Lambda]\}$, is plotted in panel (c). Plots (d), (e) and (f) display, respectively, dynamics, Fourier spectra and phase space maps of the signals obtained for two values of $\left|\Omega_{0}\right|$ shown in the plots. For $\left|\Omega_{0}\right|=200 \gamma_{31}$, the dynamics demonstrates selfoscillations, while for $\left|\Omega_{0}\right|=302.4 \gamma_{31}$ - (quasi)chaotic behaviour.
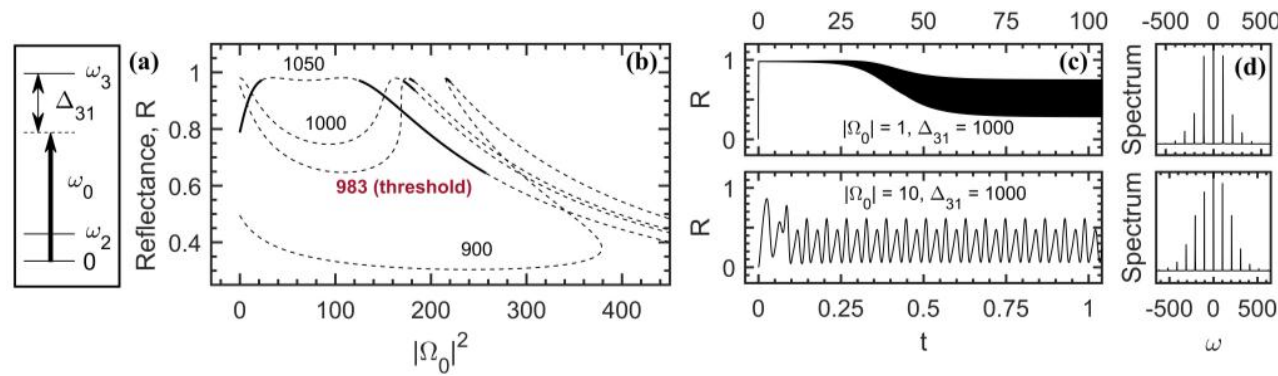

Fig. 2. (a) - Scheme of QE excitation. (b) - The steady-state layer's reflectance R calculated for a set of the detuning $\Delta_{31}$ (shown in the panel). Solid (dashed) parts of the curves indicate regions of stability (instability) of R. (c) and (d) - Dynamics and Fourier spectra, respectively, calculated for the values of $\left|\Omega_{0}\right|$ shown in the panel (c). All quantities are given in units of $\gamma_{31}$.

Fig. 2 shows the power refection coefficirnt $\mathrm{R}$ calculated in the vicinity of the resonance renormalized by the QE-QE dipole-dipole interaction, $\Delta_{31}=\Delta_{\mathrm{L}}=1000 \gamma_{31}$ [1,2]. As follows from the figure, the monolayer exhibits high reflectance and bistability [panel (b)] as well as self-oscillations [panel (c)].

Summarizing, a monolayer of $\Lambda$-type QEs demonstrates multistability, self-oscillations, dynamical chaos and high reflectance, the features being perspective for nanophotonics.

R.F.M. acknowledges Akmullah State Pedagogical University for a support.

\section{References}

1. R.F. Malikov, V.A. Malyshev, Opt Spectrosc. 122, 955 (2017)

2. V.A. Malyshev et al., J. Phys.: Conf. Ser. 1220, 012006 (2019)

3. M.P. Boneschanscher, W.H. Evers, J.J. Geuchies et al., Science 344, 1377 (2014) 